\title{
Supporting CSCW and CSCL with Intelligent Social Grouping Services
}

\author{
Jeffrey J.P. Tsai, University of Illinois, USA \\ Jia Zhang, Northern Illinois University, USA \\ Jeff J.S. Huang, National Central University, Taiwan \\ Stephen J.H. Yang, National Central University, Taiwan
}

\begin{abstract}
This paper presents an intelligent social grouping service for identifying right participants to support CSCW and CSCL. We construct a three-layer hierarchical social network, in which we identify two important relationship ties - a knowledge relationship tie and a social relationship tie. We use these relationship ties as metric to measure the collaboration strength between pairs of participants in a social network. The stronger the knowledge relationship tie, the more knowledgeable the participants; the stronger the social relationship tie, the more likely the participants are willing to share their knowledge. By analyzing and calculating these relationship ties among peers using our computational models, we present a systematic way to discover collaboration peers according to configurable and customizable requirements. Experiences of social grouping services for identifying communities of practice through peer-to-peer search are also reported.
\end{abstract}

Keywords: $\quad$ collaborative work; intelligent grouping; social factors; Web services

\section{INTRODUCTION}

Although the Internet technology has made it possible for people to collaborate effectively without staying physically together, they have led to the unintended consequence of increasing isolation among people with respect to their academic peers. In bygone times, the inconvenience of having to share resource sites (for example, computer centers and unscheduled laboratory use) afforded opportunities for de- veloping computer-oriented social groups for virtual collaboration.

Computer Supported Cooperative Work (CSCW) provides a virtual collaboration technology that offers participants a promising option of not being physically present at cooperation. Applied to collaborative learning, CSCW techniques allow students to study in a virtual team without physically staying at a common place (Weinberger, \& Fischer, 2006). Computer-Supported Collaborative Learning 
(CSCL) was thus coined in 1996 (Koschmann, 1996) to refer to adopting CSCW technology to provide a computer and network-supported collaborative learning platform for students to study cooperatively to acquire knowledge (Komis, Avouris, \& Fidas, 2002).

While there have been significant efforts developing collaborative learning environments for existing groups, little work has been done to help people find proper partners in Internet communities. In our vision, qualitative principles and strategies from traditional higher education research and practices should be normalized and quantified into computer understandable and interpretable rules, and guide automatic formation of cooperative groups.

This research aims to promote Internetbased informal collaboration over CSCW and CSCL, by exploring the plausibility of providing system-level support and services for the forming of collaborative groups dynamically. Our outcome will lead to a plug-in into the existing Web-based platform providing intelligent social grouping services. Based on our study and surveys, we focus on exploring how to exploit knowledge and social networks on top of historical data to help students establish subgroups of cohorts that may become "communities of practice." By communities of practice, we borrow from social science and refer to a group of participants with common interests in a particular subject. By participants, we refer to the individuals who (1) possess related information, (2) can help to discover and obtain the information, or (3) are willing to exchange and share information with others.

This paper presents an intelligent social grouping service empowered by social networkbased peer-to-peer (P2P) search to facilitate the identification and establishment of communities of practice on the Internet. Here, peers represent individuals (participants) who are associated with the communities through knowledge and social relationships. Throughout this paper, we will use the terms "peer" and "participants" interchangeably. We propose two important relationship ties, a knowledge relationship tie and a social relationship tie, as underlying metric to measure the degrees of a peer's knowledge matching and social relationships regarding a query initiated by another peer. By analyzing and calculating these relationships among peers using our computational models, we present a systematic way to discover peers based on configurable and customizable requirements. We have also conducted experiments to evaluate how our method improves the identification of communities of practice on the Interne.

The remainder of the paper is organized as follows. We first review related work in section 2. We present our knowledge and social network-based P2P search framework and the methods for calculating knowledge relationship tie and social relationship tie in section 3 . We present our system implementation and discuss our experiments and results in section 4, and finally, we draw conclusions in section 5 .

\section{RELATED WORK}

In the literature, numerous research and practices have reported a number of instructional supporting techniques that stimulate and facilitate cooperative learning (Jonassen, 2004), in both face-to-face and computer and networkbased contexts (Hron, Friedrich, 2003). Here, we concentrate on three areas most relevant to this research: CSCW and CSCL, P2P, as well as social networks.

\section{CSCW and CSCL}

Since its inception in 1984 (Grudin, Poltrock, 1997), Computer Supported Cooperative Work (CSCW) provides a virtual collaboration technology that offers participants a promising option of not being physically present at a cooperation. Applied to collaborative learning, CSCW techniques allow students to study in a virtual team without physically staying at a common place (Weinberger, \& Fischer, 2006). Computer-Supported Collaborative Learning (CSCL) was thus coined in 1996 (Koschmann, 1996) to refer to adopting CSCW technology to provide a computer and network-supported 
collaborative learning platform for students to study cooperatively to acquire knowledge (Komis, Avouris, \& Fidas, 2002). More specific, Internet-based CSCL is also referred to as Web-Based Collaborative Learning (WBCL) (Hron, Friedrich, 2003), which is the focused area of this research. Throughout this paper, we will use CSCL and WBCL interchangeably. WBCL differentiates from conventional collaborative learning from several significant perspectives such as social communication situation, message exchange, cognitive load, and participation of learners (Hron, Friedrich, 2003). Kollias and others (Kollias et al., 2005) study, from a teacher's perspective, how WBCL can complement and improve classroom study. Hron and Friedrich (Hron, Friedrich, 2003) examine beyond-technique factors of WBCL. Through the development of two WBCL environments, Rubens and others (Rubens et al., 2005) summarize a set of pedagogical principles for building WBCL systems.

A CSCL-oriented software system is usually called a Collaborative Learning Environment (CLE). Some existing CLE examples are: Conversant Media (Lourdusamy et al., 2003), COMTELLA (Vassileva, 2004), EDUCOSM (Miettinen et al., 2003), EDUTELLA (Nejdl et al., 2002), Groove (Eikemeier, Lechner, 2004), and SpeakEasy (Edwards et al., 2002). Bravo and others (Bravo et al., 2006) build a learner-centered synchronous CSCL environment for students to study design. Collaboration feedbacks are also used to enhance collaborative e-learning (Zumbach, Hillers, \& Reimann, 2003). A CLE can be established using all of the three aforementioned grouping approaches. For the formal learning groups and study teams approaches, a CLE provides a long-running virtual collaboration environment; for the informal learning groups approach, a CLE enables a dynamic way for students to group into a virtual learning team at run time.

Existing CSCL research focuses on how to enhance interactions (Chang, Zhang, Chang, 2006) within an already formed learning group (Zurita, Nussbaum, 2004) to improve communication, coordination, negotiation, and interactivity (Gutwin et al., 1996). In contrast to their work, our research focuses on how to form an effective learning group.

\section{P2P for CSCL}

A P2P network is a distributed networking structure that treats every participant as a peer and allows each peer to play as either a client or a server under different circumstances (Brase, Painter, 2004). P2P network is considered as a more suitable platform to build CSCL systems compared with traditional client-server model (Cuseo, 2002). Some specialized educational P2P applications have been developed and their experiences have been reported, such as COMTELLA (Vassileva, 2004), EDUCOSM (Miettinen et al., 2003), EDUTELLA (Nejdl et al., 2002), Groove (Eikemeier, Lechner, 2004) and SpeakEasy (Edwards et al., 2002). Manlove and others (Manlove et al., 2006) conclude from a case study that P2P-based CSCL tools promote student learning in scientific inquiry learning.

Some researchers track and analyze interaction patterns in a P2P environment to guide collaborative learning (Daradoumisa et al., 2006). For example, Avouris and others (Avouris et al., 2004) design an environment for monitoring and examining group learning patterns from two aspects - activity analysis and collaboration analysis. Some other researchers focus on building powerful P2P learning environments such as Conversant Media (Lourdusamy et al., 2003) and metacognition (Dimitracopoulou, Petrou, 2005).

Compared with these related work on P2P networks, we use the P2P technology as our underlying backbone to build a hierarchical framework supporting CSCW and CSCL. We utilize the P2P technique to build both knowledge network and social network in our framework.

\section{Social Networks}

Research results and practices from the fields such as educational communications, social 
sciences, and psychological sciences, have provided a variety of guidelines for people to dynamically form proper teams and groups. Among others, many researchers have proven that social relationships and interactions have significant impacts on collaborative learning (Zurita, Nussbaum, 2004) (Dimitracopoulou, Petrou, 2005) (Fischer et al., 2002). Fischer and others (Fischer et al., 2002) also conclude that social relationships have an impact on knowledge acquisition in a collaboration mode. The technique of social network is thus used to represent a determinable networking structure of how people know each other (Raghavan, 2002) (Kautz et al., 1997) (Alani et al., 2003). A social network can be formalized into a net structure comprising nodes and edges. In such a network, nodes represent individuals or organizations. Edges connecting nodes are called ties, which represent the relationships between the individuals and organizations, either directly or indirectly (Churchill, Halverson, 2005). The strength of a tie (weight of an edge) indicates the strength of the relationship.

Many kinds of ties may exist between the nodes in a social network (Churchill, Halverson, 2005). One popular tie is social interaction tie, which refers to the structural links created through social interactions between individuals in a social network (Wasko, Faraj, 2005) (Zhang, Jin, \& Lin, 2005). Prior studies suggest that an individual's centrality in an electronic network of practice can be measured using the number of social ties that an individual has with others in the network (Ahuja et al., 2003). Tsai and Ghoshal (Tsai, Ghoshal, 1998) report that social interaction tie has positive impacts on the extent of inter-unit resource exchange. Wasko and Faraj (Wasko, Faraj, 2005) discover that the centrality established by the social interaction ties significantly impacts the helpfulness and the volume of knowledge contribution. Ahuja and others (Ahuja et al., 2003) suggest that an individual's centrality in an electronic network of practice be measured using the number of social ties that an individual has with others in the social network. Kreijns and others (Kreijns et al., 2005) conclude that social interactions largely affect group forming and group dynamics.

Compared with related work, we construct a social network as an integral layer of our hierarchical framework to search for potential collaborators. We exploit existing research results in the field of social network to design questionnaires to identify decision factors for calculating social interaction ties.

\section{Social Network-based P2P Search for Intelligent Grouping}

The intelligent social grouping service is empowered by a social network-based P2P search. In this research, we found P2P and social networks share many concepts in common. For example, they are both distributed networking structures; a peer in a P2P network can be viewed as an analog of a node in a social network; a link in a P2P can be viewed as an analog of a relationship tie in a social network. In contrast to most $\mathrm{P} 2 \mathrm{P}$ researches that emphasize on search queries and protocols, our social network-based P2P search aims at reducing search time and decreasing message traffic by minimizing the number of messages circulating in the network.

\section{Decision Factors}

At the beginning of the project, we conducted a survey to identify a set of initial decision factors for group forming. We chose to recruit out of the Computer Science freshman class, so that the selected subjects possess overlapping knowledge backgrounds over generic study topics.

The survey was processed in two phases. In phase 1, a study topic was announced in the class. Students were asked to describe, in a verbose manner, how they would like to choose study partners on the Internet for the predefined study topic. They were asked to focus on the decision factors on which they form study groups and the key criteria of how they define "effective groups." After gathering students' answer sheets, we summarized and abstracted 
a set of decision factors and criteria. In phase 2 , each student was asked to rank the obtained factors and criteria.

As shown in Figure 1, our survey revealed that students essentially count on two key factors to form a study team: knowledge possession and social relationship. The former indicates whether a student possesses relative knowledge; the latter indicates whether the student may be willing to participate. For example, a student prefers to find a partner who has some knowledge about the study topic than someone who knows nothing about the topic. Also, a student prefers to have his/her friend as a partner. In turn, we found the students ranked high the following three knowledge relationship factors: knowledge domain, proficiency, and reputation of contribution. We also found that students ranked high the following two social relationship factors: social familiarity and social reputation.

\section{Social Network-based P2P Framework}

Based on our survey results, we decide to focus on two relationship ties on a P2P network: knowledge relationship tie and social relationship tie. A knowledge tie represents the degree of how a peer is familiar with the knowledge requested by an initiative peer. A relationship tie represents the degree of social relationship between a pair of involved peers. For simplicity, in this research we consider the social relationship between a pair of peers as a mutual relationship. In other words, in our social network is represented in an un-directional graph.

Based on the two relationship ties, we established a social network-based P2P framework for supporting group formation. As shown in Figure 2, our framework comprises three layers: a P2P knowledge net (K-net), a P2P social net (S-net), and an IM-equipped group discussion layer. Note that all users having access to the Internet form a P2P network space. Conceptually, each layer contains a P2P network, with each node denoting a peer in the P2P network space. In the first and the lowest K-net layer, an edge represents a knowledge relationship tie. In the second S-net, an edge represents a social network tie. In the third layer, all nodes represent the search results of potential collaborators.

Our framework also shows how to dynamically create the three layers to support

Figure 1. Decision factors

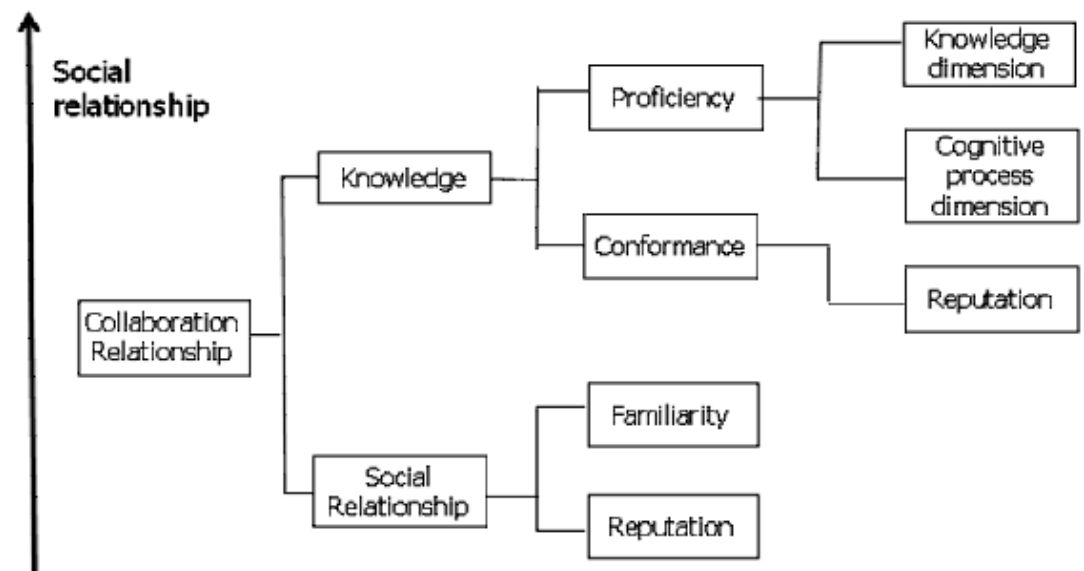

Knowledge relationship

Copyright (C) 2009, IGI Global. Copying or distributing in print or electronic forms without written permission of IGI Global is prohibited. 
smart group formation. The idea is illustrated in Figure 2. For a given query requesting participants with certain knowledge, a group of potential collaborators (with strong social relationships with the initiative peer and with the requested knowledge) will be found. We will use an example shown in Figure 2 to walk readers through.

Assume that peer Jeff initiates a request searching for peers with knowledge about "Software Engineering." For Jeff's request, a $\mathrm{P} 2 \mathrm{P} \mathrm{K}$-net is dynamically generated. Available peers in the original $\mathrm{P} 2 \mathrm{P}$ network space are examined against the requested knowledge. An edge is drawn from the requester peer to every tested peer, with a number assigned as the value of a knowledge tie. How we quantify a peer's familiarity with the requested knowledge can refer to our previous report (Yang et al., 2007). As shown in Figure 2, peers Chris and Albert hold knowledge relationship ties $(0.8)$ and $(0.16)$ with Jeff, respectively. This means that Chris knows more about "Software Engineering" than Albert does. A threshold is predefined, say 0.5 , to facilitate social grouping. Peers on the K-net with knowledge ties lower than the threshold will be removed from the K-net without further considerations (for example, peer Mary). Finally, the K-net contains peers who know enough about the knowledge. These peers form into a pool of active peers.

Then we construct a P2PS-net, as shown in Figure 2. We calculate the social relationship tie between each pair of the peers from the K-net. Our algorithm to calculate a social relationship tie will be discussed in section 4. As shown in Figure 2, Jeff is more familiar with Albert than Chris because the social relationship tie between Jeff and Albert is (0.9), which is greater than that between Jeff and Chris (0.8). Peers appear on S-net with negative relationships with the requester are removed (for example, $B o b$ ). Specific rules may be defined to decide whether all involved peers have to meet a curtain level of social relationship. For example, whether any pair of peers left cannot have negative social relationship ties between them.
The resulted peers in S-net (Chris and Albert) are potential collaborators for Jeff. As IM-based group discussion is one way of social collaboration toward sharing explicit and tacit knowledge, Jeff initiates an IM-equipped group chatting, as shown as the third layer in Figure 2. This example shows that the essential challenge of constructing this three-layer framework is how to calculate the knowledge relationship tie and social relationship tie.

\section{Calculating Knowledge Relationship Tie between Peers}

Based on our survey, as shown in Figure 1, we consider a peer's knowledge domain, proficiency, and reputation of contribution as key indicators determining its capability to participate in collaborations. Therefore, as shown in the P2P K-net in Figure 2, we calculate a peer's knowledge relationship tie based on these three indicators.

We apply Bloom taxonomy matrix (Anderson et al., 2001) to classify a peer's domain knowledge and its proficiency in such a domain. Bloom taxonomy is a two-dimensional matrix containing Knowledge dimension and Cognitive Process dimension. The former indicates the types of knowledge; the latter indicates cognitive processing of knowledge. Each cell in the matrix is associated with a value ranging from 0 to 1 , representing the level of proficiency. We adopt similar mechanism to represent a peer's reputation regarding a specific knowledge domain.

Consider peer $i$ on a P2P network is requesting peer $j$ whose knowledge proficiency conforms to a requested knowledge domain $k$. Peer $i$ 's query can be calculated by:

$$
\begin{aligned}
& K_{(k)}^{\text {tie }}(i, j)= \\
& K_{(k)}^{\text {proficiency }}(j) \bullet K_{(k)}^{\text {conformance }}(i)^{T} \bullet K_{(k)}^{\text {reputation }}(j)
\end{aligned}
$$

where

$K_{(k)}^{t i e}(i, j)$ is a real number between 0 and 1 , representing the knowledge relationship tie 
Figure 2. A three-layer social network-based P2P framework

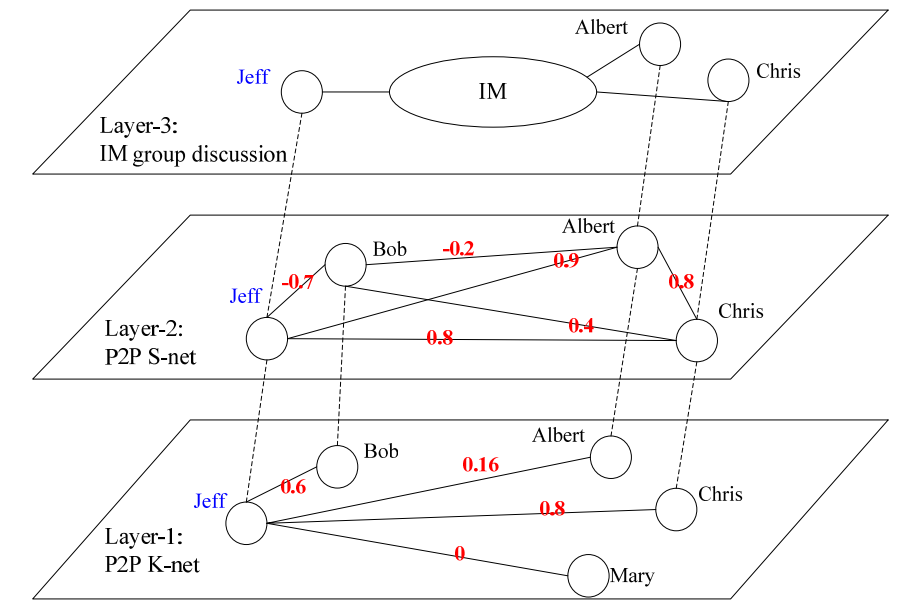

between peer $i$ and peer $j$ w.r.t. knowledge domain $k$.

$K_{(k)}^{\text {proficiency }}(j)$ is a Bloom taxonomy matrix representing peer $j$ 's knowledge proficiency w.r.t. a requested knowledge domain $k$.

$K_{(k)}^{\text {conformance }}(i)$ is a Bloom taxonomy matrix representing a conformance requirement requested by peer $i$ to peers whose knowledge proficiency conforms to a requested knowledge domain $k$.

$K_{(k)}^{\text {reputation }}(j)$ is a real number between 0 and 1 , representing peer $j$ 's reputation regarding contribution to the requested knowledge domain $k$.

\section{Calculating Social Relationship Tie between Peers}

A social relationship tie indicates the degree of social relationships between a pair of peers on the P2P S-net. For a pair of peers, denoted by peer $i$ and peer $j$, we define the social relationship tie between them as the product of their social familiarity and social reputation.

$S^{\text {tie }}(i, j)=S^{\text {familiarity }}(i, j) \times S^{\text {reputation }}(j)$

where,
$S^{t i e}(i, j)$ is a social relationship tie between peers $i$ and $j$, $S^{\text {familiarity }}(i, j)$ is a social familiarity between peers $i$ and $j$, $S^{\text {reputation }}(j)$ is peer $j$ 's social reputation.

Social familiarity indicates the level of familiarity (for example, ranging from casual to close) between two peers. When a new peer connects to a social network, every existing peer will be notified and needs to specify his/her social familiarity with the new peer (for example, by filling forms and answering questionnaires). The default value is zero, meaning that there is no relationship with the newly joined peer. Social familiarity can be exhibited in different levels, such as friends, team-mates, organization colleagues, or virtual community members. Meanwhile, social familiarity can be either positive or negative values ranging between -1 and 1, indicating the relationship is either good or bad. To perform quantitative analysis, without losing generality, we define social familiarity between peers $i$ and $j$ into nine categories, represented by a lookup table (see Table 1).

Each peer has a social reputation, which is the product of the peer's social rating (Jones, Issroff, 2005) and the average of the peer's social familiarities. Social reputation represents 
Table 1.

\begin{tabular}{|l|l|}
\hline$S^{\text {familiarity }}(i, j)$ & Implications \\
\hline 0 & there is no relationship between peer $i \& j$ \\
\hline $0 \sim 0.2$ & peer $i$ considers peer $j$ a virtual community member with a positive relationship \\
\hline $0.3 \sim 0.4$ & peer $i$ considers peer $j$ an organization colleague with a positive relationship \\
\hline $0.5 \sim 0.7$ & peer $i$ considers peer $j$ a team-mate a with positive relationship \\
\hline $0.8 \sim 1.0$ & peer $i$ considers peer $j$ a friend with a positive relationship \\
\hline$-0.8 \sim-1.0$ & peer $i$ considers peer $j$ a friend with a negative relationship \\
\hline$-0.5 \sim-0.7$ & peer $i$ considers peer $j$ a team-mate with a negative relationship \\
\hline$-0.3 \sim-0.4$ & peer $i$ considers peer $j$ an organization colleague with a negative relationship \\
\hline $0 \sim-0.2$ & peer $i$ considers peer $j$ a virtual community member with a negative relationship \\
\hline
\end{tabular}

a degree of confidence to a target peer from all other peers on a social network who know the target peer. The social reputation of peer $j$ is computed as follows:

$$
\begin{aligned}
& S^{\text {reputation }}(j) \\
& =A V G\left[S^{\text {familiarity }}(j)\right] \times S^{\text {rating }}(j) \\
& =\frac{\sum_{m=1}^{N o P(m)}\left[S^{\text {familiarity }}(j, m)\right]}{N o P(j)} \times S^{\text {rating }}(j)
\end{aligned}
$$

where,

$A V G\left[S^{\text {familiarity }}(j)\right]$ is an average value of peer $j$ s social familiarities,

$S^{\text {rating }}(j)$ is peer $j$ 's social rating,

$N o P(j)$ is the number of peers connected to peer $j$.

\section{System Implementation and Experiments}

We have developed a $\mathrm{P} 2 \mathrm{P}$ prototype, called SOtella as shown in Figure 3. Utilizing our social network-based P2P search, SOtella can form intelligent social grouping based on knowledge relationship tie and social relationship tie. We also conducted experiments for evaluating how well our method can facilitate intelligent social grouping.

\section{P2P System Implementation}

SOtella is implemented based on open-source software, Edutella (http://edutella.jxta. org/), which is an academic P2P framework equipped with a Resource Definition Framework (RDF)-based metadata to enhance resource description and discovery. To control the experiment scale and monitor SOtella's performance, we confine the search scope of SOtella to a small-scale network including about 56 peers within a university, from a class in Department of Computer Science. Each peer in SOtella is associated with knowledge and social relationship ties as presented in the aforementioned framework.

The implementation result of the aforementioned social grouping is shown in Figure 3. The upper left part of Figure 3 shows a list of collaborative social groups categorized by their study topics. The lower left part shows the group search interface, where a peer can search using a combination of keyword, study topic, person name, email address, and organization. The right part of Figure 3 shows that a peer may browse the detail of a selected study group.

To enable adaptive group formation, we realized a rating mechanism (Jones, Issroff, 
Figure 3. Screen shot of SOtella

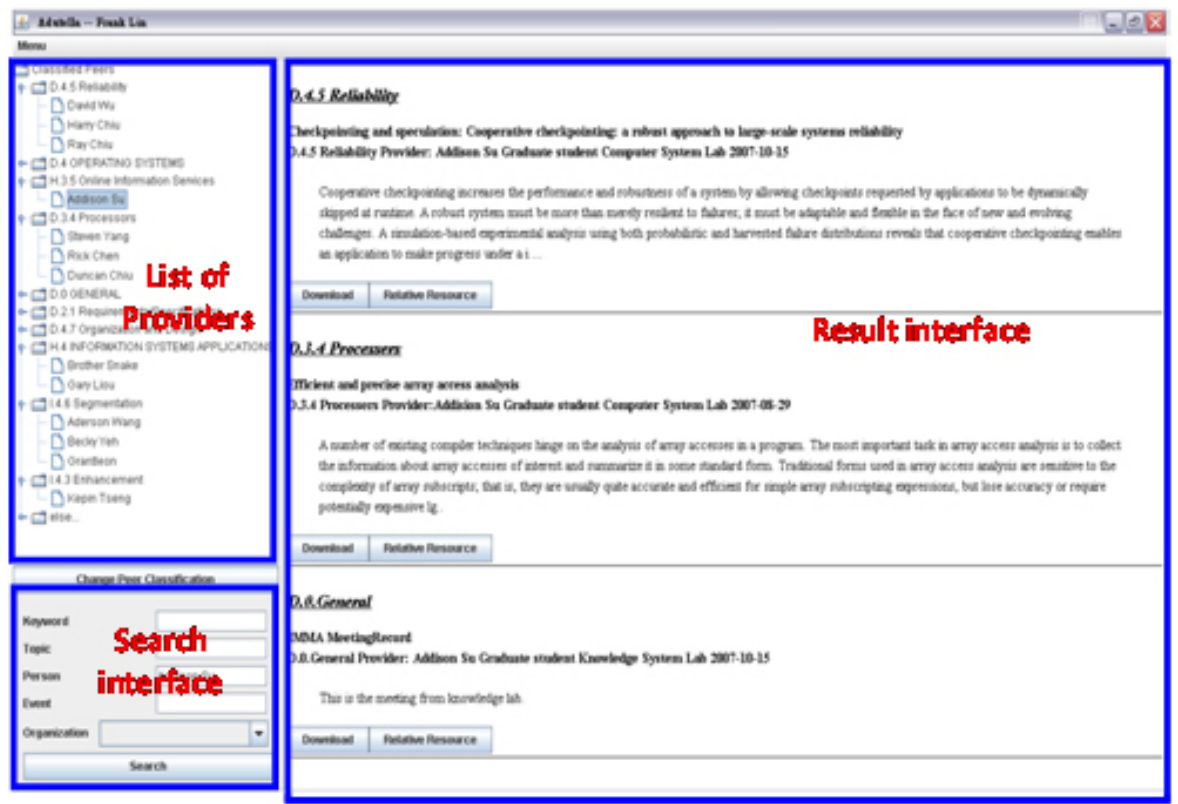

2005). Peers may provide feedbacks to the social network by the rating mechanism, so that the calculation of knowledge and social relationship ties may be further improved. For example, after a successful collaboration, a peer may change his/her social familiarity with another peer in the formed group. This updated information can be stored and used to support future group formation.

Once potential collaborators are found, we build supporting software to facilitate collaboration. As a proof of concept, we built an IM-enabled group discussion tool. Its core component is a real-time discussion board, as shown in Figure 4. Peers can communicate with collaborators relevant to their needs and improve collaboration through such a group discussion.

As shown in Figure 4, our group discussion tool provides a roster list for each participant (Albert and Chris in this example) showing who are involved in the formed group. Participants are notified when other group members get online and can review their profiles. A partici- pant may start discussions with a specific group member or initiate group discussions.

Our tool supports hand-writing annotation as well as voice-based annotation for text-based discussion. As shown in Figure 4, Chris asks Albert to explain the diagram, using hand-writing annotation. Albert sends back hand-writing annotation to point to the diagram and associate with a recorded annotation.

\section{EXPERIMENTS AND DISCUSSIONS}

After building the prototype system, we designed and conducted a pilot study of qualitative experiment to evaluate how well the intelligent social grouping services can be realized by our social network-based P2P framework. To understand the degree of users' satisfactions to the identified communities of practice, we arranged interviews at the end of the project for participating students to get direct and open-ended responses about how the prototype 
Figure 4. Screen shots of IM-equipped group discussion

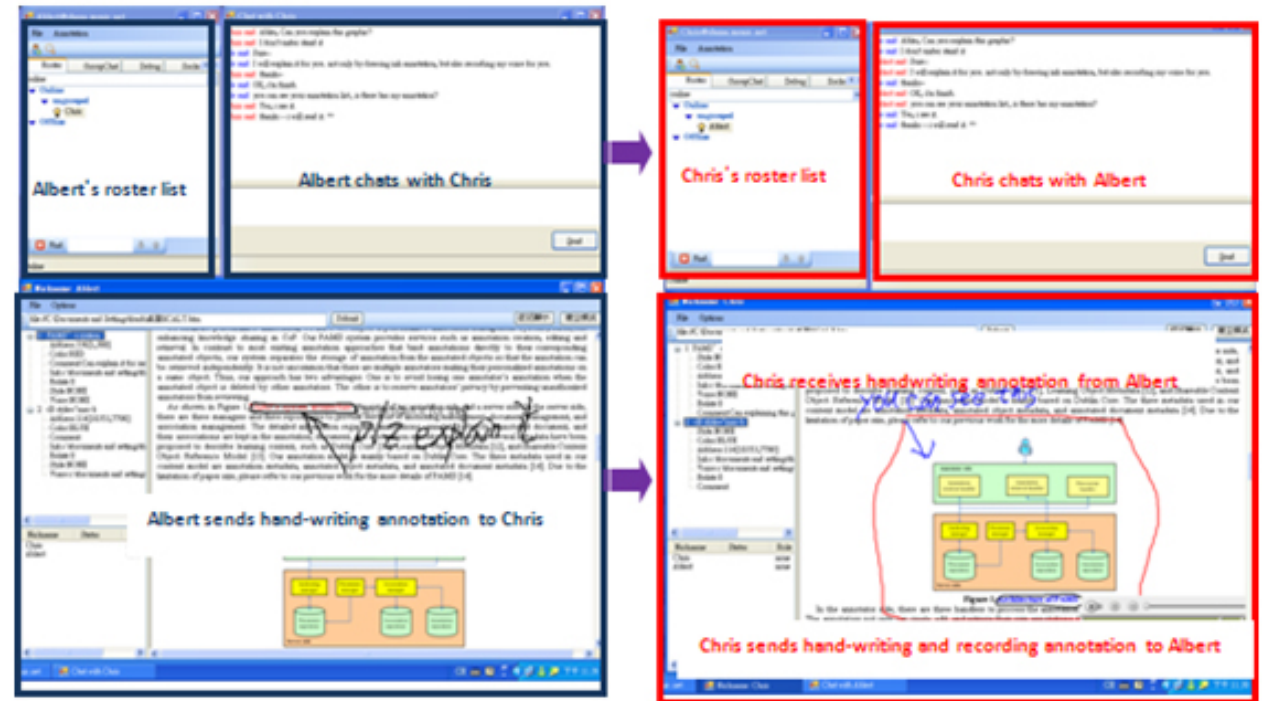

works. Every participating student was asked to complete a questionnaire to measure his/her satisfaction levels with our social network-based P2P framework.

The survey reveals five findings. First, most of the participants whom found by SOtella match students'needs in terms of knowledge and social relationships. Second, students reported that even the same search option may yield different results in different trials. We found the reason is that our P2P network only searches for participants currently on line. This symptom can be alleviated since the survey shows that most of the students remain on line most of the time. Third, we found that most of the students were satisfied with the automatic identification of communities of practice. However, they still preferred to find their own participants, even though they admitted that the communities of practice identified by SOtella were knowledgeable and close to their needs. This observation suggested that we should take into account students' autonomy in addition to knowledge competence when we identify communities of practice. Fourth, most of the students emphasized the importance of user interface design of social communication and collaboration. Fifth, we perceived that students desired powerful social networking software, such as Blogs, Wikis, RSS feeds, and video podcast for synchronous discussion and file sharing.

In summary, our experiment confirmed the effectiveness of utilizing our social networkbased P2P search method for the identification of social groups as a Web service. Most students expressed their willingness to utilize SOtella for their daily studies.

\section{CONCLUSION}

The major contribution of this paper is applying social network technique to improve P2P search by finding knowledgeable and socially related participants to form social groups in the CSCW and CSCL context. In this paper, we have presented a three-layer social network-based P2P framework equipped with the calculation methods of knowledge relationship tie and social relationship tie. Through such a framework, we demonstrated a new possibility of using social network to enhance P2P so that query can be 
routed to peers with strong relationship ties. Theories and models developed in support of the prototype system will also contribute to our general understanding and guide the creation of collaborative CSCW and CSCL.

We see several areas that deserve further research. Peers may have their own needs when they search for participants and interact with others; therefore, we need to conduct further study on new relationship ties and investigate special requirements from different social perspectives in addition to knowledge and social relationships.

\section{REFERENCES}

Ahuja, M., Galletta,D. and Carley, K. (2003). Individual Centrality and Performance in Virtual R\&D Groups: An Empirical Study. Management Science, 49(1), 21-38.

Alani, H., Dasmahapatra, S., O’Hara, K. and Shadbolt, N. (2003). Identifying Communities of Practice through Ontology Network Analysis. IEEE Intelligent Systems, 18(2), 18-25.

Anderson, L.W., Krathwohl, D.R., Airasian, P.W., Cruikshank, K.A., Mayer, R.E., Pintrich, P.R., Raths, J. and Wittrock, M.C. (2001). A Taxonomy for Learning, Teaching, and Assessing: A Revision of Bloom's Taxonomy of Educational Objectives. New York, Longman.

Andrieux, A., Czajkowski, K., Dan, A., Keahey, K., Ludwig, H., Nakata, T., Pruyne, J., Rofrano, J., Tuecke, S. and Xu, M. "Web Services Agreement Specification(WS-Agreement). 2005, Sep. 20, 2005; Available from: http://www.ggf.org/Public Comment_Docs/Documents/Oct-2005/WS-AgreementSpecificationDraft050920.pdf.

Avouris, N., Komis, V., Margaritis, M. and Fiotakis, G. (2004). An Environment for Studying Collaborative Learning Activities. Educational Technology \& Society, 7(2), 34-41.

Bravo, C. M., Redondo, A., Ortega, M. and Verdejo, M.F. (2006). Collaborative Environments for the Learning of Design: A model and A Case Study in Domotics. Computers \& Education, 46, 152-173.

Brase, J. and Painter, M. (2004). Inferring Metadata for a Semantic Web Peer-to-Peer Environment. Educational Technology \& Society, 7(2), 61-67.

Chang, C.K., Zhang, J. and Chang, K.H. (2006). Survey of Computer Supported Business Collaboration in Support of Business Processes. International Journal of Business Process Integration and Management (IJBPIM), 1(2), 76-100.

Churchill, E.F. and Halverson, C.A. (2005). Social Networks and Social Networking. IEEE Intelligent Systems, 20(5), 14-19.

Cuseo, J.B.(2002). Igniting Student Involvement, Peer Interaction and Teamwork. New Forums Press Inc., Stillwater, Oklahoma, USA.

Daradoumisa, T., Martı'nez-Mone's, A. and Xhafa, F. (2006). A Layered Framework for Evaluating On-Line Collaborative Learning Interactions. International Journal of Human-Computer Studies, 64, 622-635.

Dimitracopoulou,A. and Petrou,A. (2005).Advanced Collaborative Distance Learning Systems for Young Students: Design Issues and Current Trends on New Cognitive and Metacognitive Tools. Education International Journal, 4(11), 214-224.

Edwards, W.K., Newman, M.W., Sedivy, J.Z., Smith, T.F., Balfanz, D., Smetters, D.K., Wong, H.C. and Izadi, S. (2002). Using Speakeasy for ad hoc Peer-toPeer Collaboration. in Proceedings of the 2002 ACM Conference on Computer Supported Cooperative Work (CSCW), New Orleans, LO, USA, 256 -265.

Eikemeier, C. and Lechner, U. (2004). Peer-to-Peer and Group Collaboration - Do They Always Match?. in Proceedings of 13th IEEE International Workshops on Enabling Technologies: Infrastructure for Collaborative Enterprises (WET ICE), Jun, 101-106.

Fischer, F., Bruhn, J., Gräsel, C. and Mandl, H. (2002). Fostering Collaborative Knowledge Construction with Visualization Tools. Learning and Instruction, $12,213-232$.

Grudin, J. and Poltrock, S. (1997) Computer-supported cooperative work and groupware. Advances in Computers, 1997, 45, 269-320.

Gutwin, C., Roseman, M. and Greenberg, S. (1996). AUsability Study of Awareness Widgets in A Shared Workspace Groupware System. in Proceedings of the 1996 ACM conference on Computer Supported Cooperative Work (CSCW), Boston, MA, USA, 258-267. 
Hron, A.and Friedrich, H.F. (2003). A Review of Web-Based Collaborative Learning: Factors Beyond Technology. Journal of Computer Assisted Learning, 19, 70-79.

Jonassen, D.H. (2004). Handbook of Research on Educational Communications and Technology: A Project of the Association for Educational Communications and Technology, 2nd. ed., Mahwah, NJ, USA: Lawrence Erlbaum Associates Inc.

Jones, A. and Issroff, K. (2005). Learning Technologies: Affective And Social Issues in ComputerSupported Collaborative Learning. Computers \& Education, 44, 395-408.

Kautz, H., Selman, B. and Shah, M. (1997). ReferralWeb: Combining Social Networks and Collaborative Filtering. Comm. of ACM, 40(3), 27-36.

Kollias, V., Mamalougos, N., Vamvakoussi, X., Lakkala, M. and Vosniadou, S. (2005). Teachers' Attitudes to and Beliefs about Web-Based Collaborative Learning Environments in the Context of An International Implementation. Computers \& Education, 45, 295-315.

Komis, V., Avouris, N. and Fidas, C. (2002). Computer-Supported Collaborative Concept Mapping: Study of Synchronous Peer Interaction. Education and Information Technologies, 7(2), 169-188.

Koschmann, T. (1996). Paradigm Shifts and Instructional Technology: An Introduction, in T. Koschmann (ed.), CSCL: Theory and Practice of An Emerging Paradigm. Lawrence ErlbaumAssociates: Mahwah, NJ, USA.

Kreijns,K., Kirschner, P.A., W Jochems,. Buuren, H.v. (2005). Measuring Perceived Sociability of Computer-Supported Collaborative Learning Environments. Computers \& Education, (in press).

Lourdusamy, A., Khine, M.S. and Sipusic, M. (2003). Collaborative Learning Tool for Presenting Authentic Case Studies and Its Impact on Student Participation. Journal of Educational Technology Systems, 31(4), 381-392.

Manlove, S., Lazonder, A.W. and Jong, T. (2006). Regulative Support for Collaborative Scientific Inquiry Learning. Journal of Computer Assisted Learning, 87-98.

Miettinen, M., Kurhila, J., Nokelainen, P., Floren, P. and Tirri, H. (2003). EDUCOSM - Personalized
Writable Web for Learning Communities. in Proceedings of International Conference on Information Technology Institute of Electrical and Electronics Engineers, 37-42.

Nejdl, W., Wolf, B., Qu, C. S., Decker, M. Sintek, A. (2002). Naeve, M. Nilsson, M. Palmer, and T. Risch. "EDUTELLA: A P2P Networking Infrastructure Based on RDF. in Proceedings of the ACM 11th international conference on World Wide Web $(W W W)$, Honolulu, Hawaii, USA, 604-615.

Raghavan, P. (2002). Social Networks: From the Web to the Enterprise. IEEE Internet Computing, 6(1), 91-94.

Rubens, W., Emans, B., Leinonen, T., Skarmeta, A.G. and Simons, R.-J. (2005). Design of Web-Based Collaborative Learning Environments. Translating the Pedagogical Learning Principles to Human Computer Interface. Computers \& Education, 45, 276-294.

Tsai, W. and Ghoshal, S. (1998). Social Capital And Value Creation: The Role Of Intrafirm Networks. Academy of Management Journal, 41(4), 464-476.

Vassileva, J. (2004). Harnessing P2P Power in the Classroom. Intelligent Tutoring Systems (ITS'2004), Lecture Notes in Computer Science 3220, 305314.

Wasko, M.M. and Faraj, S. (2005). Why Should I Share? Examining Social Capital And Knowledge Contribution in Electronic Networks Of Practice. MIS Quarterly, 29(1), 35-57.

Weinberger, A. and Fischer, F. (2006). A Framework to Analyze Argumentative Knowledge Construction in Computer-Supported Collaborative Learning. Computers \& Education, 46, 71-95.

Yang, S.J.H., Zhang, J. and Chen, I.Y.L. (2007). Web 2.0 Services for Identifying Communities of Practice through Social Networks. in Proceedings of IEEE International Conference on Services Computing (SCC 2007), Salt Lake City, UT, USA, 130-137.

Zumbach, J., Hillers, A. and Reimann, P. (2003). Supporting Distributed Problem-Based Learning: the Use of Feedback in Online Learning. in T. Roberts (ed.), Online Collaborative Learning: Theory and Practice. Idea, Hershey, PA, USA, 86-103.

Zurita, G. and Nussbaum, M. (2004). Computer Supported Collaborative Learning using Wirelessly Interconnected Handheld Computers. Computers \& Education, 42, 289-314. 
Zhang, G. Jin, Q. and Lin, M. (2005). A Framework ff Social Interaction Support for Ubiquitous Learning. in Proceedings of the 19th International Conference on Advanced Information Networking and Applications (AINA'05)

Jeffrey J.P. Tsai received his PhD degree in computer science from the Northwestern University. He is now a Professor of the Department of Computer Science at the University of Illinois, Chicago. He is also a Senior Research Fellow at the University of Texas at Austin. Tsai coauthored 10 books and has published over 230 journal articles and conference papers. Tsai is currently the co-editor-in-chief of the International Journal on Artificial Intelligence Tools and an associate editor of the IEEE Transactions on Services Computing. He was an associate editor of IEEE Transactions on Knowledge and Data Engineering, and sits on many editorial boards of international journals. His current research interests include sensor networks, ubiquitous computing, trustworthy computing, intrusion detection, software reliability and adaptation, knowledge-based software engineering, distributed real-time systems, multimedia systems, and intelligent agents. He is a Fellow of the IEEE.

Jia Zhang received her PhD in computer science from University of Illinois at Chicago in 2000. She is now an assistant professor of Department of Computer Science at Northern Illinois University. Zhang has published 1 book titled "Service Computing" and over 80 technical papers in journals, book chapters, and conference proceedings. She is an associate editor of the IEEE Transactions on Services Computing (TSC), International Journal of Web Services Research (JWSR), and the Advances in Web Services Research (AWSR) Book Series, Idea Group. Zhang serves as program vice chair of IEEE International Conference on Web Services (ICWS 2008, 2007 \& 2006). Her current research interests center around Services Computing. She is a member of the IEEE.

Jeff J.S. Huang is a PhD student at the Department of Computer Science and Information Engineering, National Central University, Taiwan. His research interests include Web 2.0, CSCW, and CSCL.

Stephen J.H. Yang received his PhD degree in computer science from the University of Illinois at Chicago in 1995. He is now a professor of the Department of Computer Science and Information Engineering and the Associate Dean of Academic Affairs, National Central University, Taiwan. He is the co-founder and the CEO of T5 Corp, a company providing XML-based Web services. Dr. Yang has published 2 books and over 150 journal articles and conference papers. He is currently the co-editor-in-chief of the International Journal of Knowledge Management \& E-Learning, and in the advisory board of the Educational Technology and Society; and in the editorial boards of the International Journal of Web Services Research and the International Journal of Knowledge and Learning. He severed as the program co-chair of IEEE MSE 2003, IEEE CAUL 2006, and IEEE W2ME 2007. His research interests include Web services, Web 2.0, software engineering, knowledge engineering, semantic Web, and context aware ubiquitous computing. He is a member of IEEE. 